\title{
Analyses of the Duration times of COVID-19 Epidemic at Various Time-Points in 11 Severely Affected Countries
}

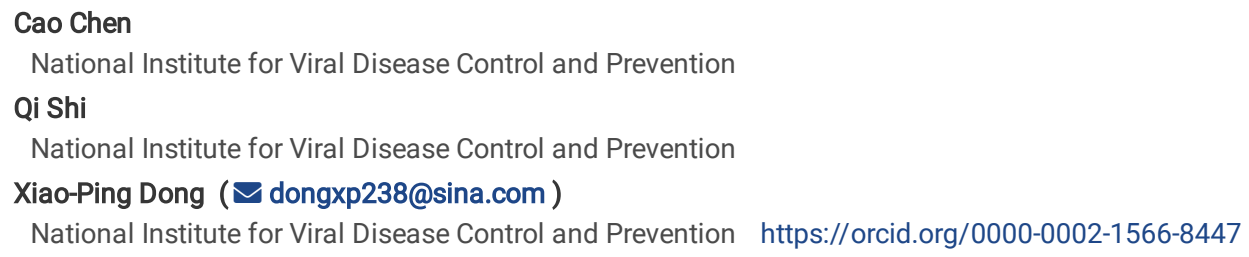

\section{Research Article}

Keywords: COVID-19, daily cases, duration time, Rt value

Posted Date: July 24th, 2020

DOI: https://doi.org/10.21203/rs.3.rs-45425/v1

License: () (i) This work is licensed under a Creative Commons Attribution 4.0 International License. Read Full License 


\begin{abstract}
Background COVID-19 pandemic has affected more than 200 different countries and territories and caused more than 4.5 million infected cases and 310,000 deaths worldwide.
\end{abstract}

Methods We collected the daily reported infected cases in 11 mostly affected countries from governmental and professional websites, including China, South Korea, France, USA, Italy, Germany, Spain, UK, Iran, Turkey and Russia. The duration times of the epidemic from the day with more than 100 cases (>100) to the day with less than 100 cases $(<100)$ in the individual countries were comparably analyzed based on the alterations of the numbers and ratios of the daily reported cases. Meanwhile, the daily Rt values each country were separately calculated.

Results We found that the duration times of decreasing period were much longer than that of increasing period. Compared with other countries, China and South Korea showed remarkably short duration times, which was likely associated with the strictly implementation of the control measures. The Rt values of all tested countries reduced to around 1.0 on the day with the largest number of cases.

Conclusion The alteration of the daily Rt value seems to be related more closely with the changes of the ratios of daily cases, rather than the absolute numbers of daily cases in one country, meanwhile the implementation of strictly tailored control measures will greatly reduce the duration of the COVID-19 epidemic.

\title{
Introduction
}

The pandemic of COVID-19 has being affected more than 200 different countries and territories worldwide since Jan 2020, causing more than 4.3 million infected and roughly 300,000 deaths by the middle of May. 10 countries reported more than 100,000 infected cases and 36 countries reported more than 10,000 cases. The crude case fatal rate (CFR) is estimated as $6.7 \%$ globally (1). The epi-wave of COVID-19 appeared in Northeast Asia, mainly in China, South Korea and Japan, from January to the middle of March, and were immediately replaced by the second but much larger wave that started from Middle East and Western Europe and soon spread to North America. Since the end of March, the infected cases were increased rapidly in the rest regions of world, such as South Asia, South America and Africa ${ }^{(1,2)}$.

By the beginning of May, only a few countries seem to be close to the late stage of epidemic with a few, even no, daily new case reported, such as China, South Korea, Australia, New Zealand, etc ${ }^{(1)}$. The daily new cases in many Western European countries appear a stable declining trend after about 2 months of high prevalence. The epidemic situations in the countries of North America are still at the high plateau. On the other hand, majority countries, particularly in South Asia, South America and Africa, are in the increasing stage ${ }^{(1-3)}$. The pandemic of COVID-19 seems to be far from ending.

In this reported, we collected and reviewed the daily reported cases in several most affected countries during the period from the day with more than 100 cases to the day with less than 100 cases and calculated the changes of the daily effective reproduction numbers (Rt). The duration times of the epidemic in the individual countries were comparably analyzed based on the alterations of the numbers and ratios (percentage accounted for the highest case) of the daily reported cases. We found that the duration times of decreasing period in all enrolled countries were much longer than that of increasing period. Compared with other countries, China and South Korea showed remarkably short duration times at high plateau. The Rt value seems to be related with the ratios, rather than the absolute numbers, of the daily cases.

\section{Materials And Methods \\ Data sources}

Ten severely affected countries were brought into this study, including the USA, Spain, Italy, France, Germany, UK, Turkey, Iran, China and Russia. Meanwhile, the Republic of Korea (South Korea) was also included. The daily data of COVID-19 cases of each countries were collected from the websites of WHO,

European CDC, as well as the relevant governmental and other professional websites ${ }^{(1-3)}$.

\section{Calculation of the intervals based on the changes of the case numbers and percentages}

The intervals based on the changes of the daily case numbers were calculated, i.e., from the $1 \mathrm{st}$ case to $>100$ cases, from $>100$ to $>3000$ cases, from $>3000$ to the top, from the top to $<3000$ cases, from $<3000$ to $<1000$ cases and from $<1000$ to $<100$ cases. The top of daily case number each country was considered based on the data by May 4, 2020. The intervals based on the changes of the percentages of the daily new cases to that of the highest cases (top, $100 \%$ ) were also calculated, including the intervals from $>100$ cases to $25 \%, 25$ to $50 \%, 50$ to $75 \%, 75 \%$ to top, top to $-75 \%,-75 \%$ to $-50 \%,-50 \%$ to $-25 \%,-25 \%$ to $<100$ cases, respectively.

SEIR modeling

The Rt value in infectious diseases is estimated using time-dependent method. The principle is that the Rt value at time $t$ is defined as the mean of the secondary cases infected by the symptomatic cases at time $t$.

Considering the differences on time of symptom onset $(t i-t)$ ), the relative probability (pij) of cases $i$ infected by cases $j$ can be represented by probability distribution of passage interval, presenting as $w(\tau)$. This model assumes that the passage interval follows $w e i b u l /$ distribution, scale $=10.22$, shape $=3.4$.

The relative likelihood of case $i$ being infected by case $j$ is the normalized ratio of the likelihood of case $i$ being infected by case $j$ to the likelihood of case $i$ being infected by any other case $k$. 
The Rt value of case $j$ is the sum weighted by the relative probability of case $i$ being infected by case $j$ :

$$
R j=\sum_{i} P_{i j}
$$

Therefore, the Rt value is the mean of the effective reproduction number of all cases.

\section{Results}

The daily new confirmed cases of COVID-19 of 10 mostly affected countries and South Korea from the date with more than 100 cases to the date with less than 100 cases or by May 4, 2020, were collected and illustrated in Fig. 1. Only USA and Russia showed the peak of daily cases higher than 10,000. The countries with the peak of daily cases higher than 5,000 but less than 10,000 were Spain, Italy, France, Germany, UK and Turkey. China and Iran showed the peaks of daily cases higher than 3,000 cases but less than 4,000 cases. The peak of daily cases in South Korea was less than 1,000 .

Only China and South Korea whose daily new cases dropped down to less than 100 cases. The intervals between $>100$ to $<100$ cases were 46 and 42 days in China and South Korea, respectively, with the peaks of 3704 cases on the day 16 in China and 815 cases on the day 12 in South Korea (Fig. 1, Table 1). The declining intervals from top to $<100$ cases in China and Korea were both 30 days, which were markedly longer than the increasing intervals from $>100$ cases to top (16 and 12 days, respectively). By May 4, the daily cases in Germany, France and Iran reduced to less than 1,000 after around 60 days, while that of Italy, Spain and Turkey were less than 3,000 and that of the USA, UK and Russia were still higher than 3,000. As shown in Table 1, the increasing intervals from > 100 to $>1,000$ cases among the countries were from 8 (China and Spain) to 14 (Russia) days. The intervals from $>1,000$ to $>3,000$ cases differed, with the shortest of the USA ( 2 days), the longest of Iran (22 days) and 6-7 days of the majority of the European countries and China. The days from $>3,000$ cases to the top day were closely related with the highest case umbers of every countries. China and Iran had the highest daily cases of 3704 and 3186 , respectively, whose intervals were 1 and 2 days. The interval of the USA was remarkably long ( 36 days), with the highest daily case number of 44,638 , while that of Russia was 18 days with the highest daily case number of 10,632 . The intervals in the 7 European countries were $6-8$ days, with the daily peaks in the zone of $5,000-$ 10,000 .

Table 1

Duration times and Rt values in the increase and decrease period based on the daily case number

\begin{tabular}{|c|c|c|c|c|c|c|c|c|c|c|c|c|c|c|}
\hline \multirow[t]{2}{*}{ Country } & \multirow{2}{*}{$\begin{array}{l}>100 \text { cases } \\
\text { day }\end{array}$} & \multicolumn{2}{|c|}{$>1000$ cases } & \multicolumn{2}{|c|}{$>3000$ cases } & \multicolumn{2}{|l|}{ Top } & \multicolumn{2}{|c|}{$<3000$ cases } & \multicolumn{2}{|c|}{$<1000$ cases } & \multicolumn{2}{|c|}{$<100$ cases } & \multirow{2}{*}{$\begin{array}{l}\text { By May } 4 \\
\text { day }\end{array}$} \\
\hline & & day & Rt & day & Rt & day & Rt & day & Rt & day & Rt & day & Rt & \\
\hline China & 1 & 8 & 2.94 & 15 & 1.50 & 16 & 1.40 & 22 & 0.53 & 30 & 0.23 & 46 & 0.27 & - \\
\hline South Korea & 1 & - & - & - & - & 12 & 0.46 & - & - & - & - & 42 & 0.37 & - \\
\hline France & 1 & 12 & 2.85 & 19 & 2.23 & 27 & 1.10 & 46 & 0.33 & 60 & 0.78 & NA & NA & 61 \\
\hline USA & 1 & 11 & 7.26 & 13 & 5.59 & 49 & 0.95 & NA & NA & NA & NA & NA & NA & 60 \\
\hline Germany & 1 & 9 & 5.45 & 16 & 1.92 & 23 & 1.06 & 39 & 0.58 & 59 & 0.50 & NA & NA & 61 \\
\hline Italy & 1 & 11 & 4.14 & 18 & 2.02 & 25 & 0.98 & 60 & 0.70 & NA & NA & NA & NA & 69 \\
\hline Spain & 1 & 8 & 5.37 & 14 & 2.50 & 21 & 0.95 & 59 & 0.34 & NA & NA & NA & NA & 61 \\
\hline UK & 1 & 10 & 3.51 & 20 & 1.55 & 30 & 0.99 & NA & NA & NA & NA & NA & NA & 54 \\
\hline Iran & 1 & 9 & 1.86 & 31 & 0.94 & 33 & 0.78 & 35 & 0.70 & 66 & 1.10 & NA & NA & 68 \\
\hline Turkey & 1 & 9 & 3.79 & 18 & 1.50 & 25 & 0.91 & 39 & 0.58 & NA & NA & NA & NA & 48 \\
\hline Russia & 1 & 14 & 3.30 & 22 & 1.93 & 40 & 1.73 & NA & NA & NA & NA & NA & NA & 41 \\
\hline
\end{tabular}

Further, we took the data of the highest daily reported as $100 \%$ and then calculated the intervals from the emerging of the $1 \mathrm{st}$ cases to the increases of $>100$ cases, approximately $25 \%, 50 \%, 75 \%, 100 \%$ and decreases of $-75 \%,-50 \%,-25 \%$ and $>100$ cases. As shown in Fig. 2 and Table 2 , the intervals from the 1 st case to $>100$ cases in the majority of the enrolled countries ranged from 25 to 45 days. Russia showed the longest interval with 54 days, whereas Iran and Turkey were much short with 7 and 8 days, respectively. Only the daily cases in China (March 12) and South Korea (April 2) declined to less than 100 cases during the epidemic, with the similar alterative patterns. Calculation of the intervals from $>100$ cases to top and from top to $<100$ cases revealed a short increasing time and a long decreasing time. The portions of increasing and decreasing times were $34.8 \%$ and $65.2 \%$ in China, and $28.6 \%$ and $71.4 \%$ in South Korea. Compared with increasing times of China (16 days) and South Korea (12 days), those of Germany (23 days), Italy (25 days), Spain (21 days), France (27 days), Iran (33 days), UK (30 days), Turkey ( 25 days) Russia (40 days) and USA (49 days) were obviously longer, particularly in the zone of $>100$ cases to $50 \%$ (Fig. 2 , Table 2). 
Table 2

Duration times and Rt values in the increase and decrease period based on the daily case

\begin{tabular}{|c|c|c|c|c|c|c|c|c|c|c|c|c|c|c|c|c|c|c|}
\hline \multirow[t]{2}{*}{ Country } & \multicolumn{2}{|c|}{$>100$ cases } & \multicolumn{3}{|c|}{ Appr $25 \%$} & \multicolumn{3}{|c|}{ Appr $50 \%$} & \multicolumn{3}{|c|}{ Appr $75 \%$} & \multicolumn{3}{|l|}{$100 \%$} & \multicolumn{3}{|c|}{ Appr $-75 \%$} & \multirow{2}{*}{$\begin{array}{l}\text { Appr } \\
\%\end{array}$} \\
\hline & $\%$ & day & $\%$ & day & Rt & $\%$ & day & Rt & $\%$ & day & Rt & $\%$ & day & Rt & $\%$ & day & Rt & \\
\hline China & $2.8 \%$ & 1 & $25.0 \%$ & 6 & 4.18 & $50.7 \%$ & 11 & 2.03 & $74.6 \%$ & 14 & 1.60 & $100 \%$ & 16 & 1.40 & $78.3 \%$ & 22 & 0.53 & $55.6 \%$ \\
\hline $\begin{array}{l}\text { South } \\
\text { Korea }\end{array}$ & $12.3 \%$ & 1 & $28.1 \%$ & 2 & 6.59 & $62.0 \%$ & 7 & 1.68 & - & - & - & $100 \%$ & 12 & 0.46 & - & - & - & $55.0 \%$ \\
\hline France & $1.8 \%$ & 1 & $24.6 \%$ & 15 & 2.59 & $50.6 \%$ & 19 & 2.24 & NA & NA & 1.55 & $100 \%$ & 27 & 1.10 & $76.4 \%$ & 40 & 0.32 & $50.5 \%$ \\
\hline USA & $0.3 \%$ & 1 & $27.4 \%$ & 19 & 2.69 & $54.1 \%$ & 25 & 1.51 & $74.3 \%$ & 29 & 1.14 & $100 \%$ & 49 & 0.95 & $75.5 \%$ & 50 & 0.85 & NA \\
\hline Germany & $3.2 \%$ & 1 & $21.0 \%$ & 12 & 3.33 & $47.7 \%$ & 15 & 2.30 & $62.1 \%$ & 21 & 1.31 & $100 \%$ & 23 & 1.06 & $71.2 \%$ & 36 & 0.54 & $47.3 \%$ \\
\hline Italy & $2.0 \%$ & 1 & $27.4 \%$ & 13 & 3.25 & $53.3 \%$ & 18 & 2.01 & $81.2 \%$ & 23 & 1.14 & $100 \%$ & 25 & 0.98 & $72.9 \%$ & 36 & 0.80 & $46.7 \%$ \\
\hline Spain & $1.7 \%$ & 1 & $25.4 \%$ & 11 & 3.62 & $48.2 \%$ & 16 & 1.95 & $79.2 \%$ & 18 & 1.50 & $100 \%$ & 21 & 0.95 & $75.9 \%$ & 34 & 0.81 & $48.3 \%$ \\
\hline UK & $1.6 \%$ & 1 & $24.5 \%$ & 13 & 2.81 & $49.8 \%$ & 21 & 1.55 & $68.0 \%$ & 25 & 1.23 & $100 \%$ & 30 & 0.99 & $67.4 \%$ & 39 & 0.92 & $46.0 \%$ \\
\hline Iran & $3.3 \%$ & 1 & $26.2 \%$ & 7 & 2.20 & $55.3 \%$ & 27 & 1.30 & $75.0 \%$ & 29 & 1.04 & $100 \%$ & 33 & 0.78 & $71.4 \%$ & 40 & 0.66 & $49.4 \%$ \\
\hline Turkey & $1.8 \%$ & 1 & $23.2 \%$ & 9 & 3.79 & $47.1 \%$ & 16 & 1.73 & $75.1 \%$ & 21 & 1.13 & $100 \%$ & 25 & 0.91 & $70.4 \%$ & 37 & 0.58 & $50.9 \%$ \\
\hline Russia & $2.7 \%$ & 1 & $26.1 \%$ & 22 & 1.92 & $26.1 \%$ & 26 & 1.66 & $74.6 \%$ & 38 & 1.77 & $100 \%$ & 41 & 1.73 & NA & NA & NA & NA \\
\hline $\begin{array}{l}\text { Average } \\
\mathrm{Rt}\end{array}$ & & & & & 3.36 & & & 1.81 & & & 1.34 & & & 1.03 & & & 0.67 & \\
\hline
\end{tabular}

The intervals from top to $<100$ cases in China and South Korea were both 30 days. The days from top to $-50 \%$, from $-50-25 \%$, and from $-25 \%$ to $<100$ cases were 9, 6 and 15 in China, 4, 5 and 21 in South Korea, respectively, in which South Korea revealed relatively long decreasing time from $-25 \%$ to $<100$ cases than China. By May 4, the daily cases in Germany, Italy, Spain, France and Iran dropped to approximate $-25 \%$, UK and Turkey to roughly - 50\%, while that of Russia and USA were still at the high plateau. Calculating the intervals from top to $-50 \%$ and to $-25 \%$ revealed markedly long times in Germany (15 and 28 ), Iran (15 and 33), France (18 and 22), more than 30 days in Spain (30 and 39), and Italy (31 and 43), respectively. The intervals from top to $-50 \%$ in UK and Turkey were 24 and 19 days, respectively (Fig. 2).

Further, the time of the high plateau ( $75 \%$ to $-75 \%)$ of the daily cases each country was analyzed. As shown in Fig. 2, China, with the peak of 3,704 cases, lasted its high plateau for only 8 days, while Iran, with the peak of 3,186 cases, lasted for 17 days. The times of the high plateau in the countries with the peaks of daily cases ranging from 5,000 to 10,000 cases were 21 days in France (peak of 7,578 cases), 16 days in Spain (peak of 8,271 cases), 13 days in Italy (peak of 6,557 cases), 15 days in Germany (peak of 6,993 cases), 14 days in UK (peak of 8,681 cases), 16 days in Turkey (peak of 5,138 cases), respectively. The high plateau of USA lasted for 28 days with the peak of 44,638 cases, while Russia was still at the stage of increase by May 10 .

The Rt value of every day since the daily cases exceeding 100 cases each country was measured and illustrated in Fig. 1 together with the daily cases. On the day $<100$ cases, the Rt values of China and Korea were 0.2655 and 0.3672 . By May 4 , the Rt values of France, Germany, Italy, Spain, UK, Iran, Turkey and USA were below 1.0 but mostly still higher than 0.5 , while that of Russia (1.7096) was still higher than 1.0 (Table 2).

The Rt values of the days of $25 \%, 50 \%, 75 \%, 100 \%,-75 \%,-50 \%$ and $-25 \%$ each country were further collected and summarized in Table 2 . Generally, the Rt values of all enrolled countries declined along with the decreases of the daily new cases. Most of the enrolled countries showed the Rt values around 1.0 on the day of the top. The Rt values of three time-points in the increase period in every country were all higher than 1.0, with the average of 3.36 on the day of $25 \%, 1.81$ on that of $50 \%$ and 1.34 at that $75 \%$. On the contrary, the Rt values of three time-points in the decrease period were lower than 1.0 , with the average of 0.67 on the day of $-75 \%, 0.57$ on that of $-50 \%$ and 0.51 on that of $-25 \%$. The Rt values of 11 countries at every time-point seemed to be quite similar that varied in a limited range.

\section{Discussion}

As a newly emerging infectious disease, COVID-19 spreads much faster and much more extensively worldwide than its siblings such as SARS and MERS (4, 5). In this report, we have comparatively analyzed the durations based on the alterations of daily reported cases of 11 mostly affected countries. Notably, the duration times of decrease periods (from top to $<100$ cases) are much longer than that of increase periods (from $>100$ cases to top). The lasting times of high plateau (from 75 to $-75 \%$ ) of China and South Korea were much shorter than those of the rest countries.

Among the 11 analyzed countries, the daily cases of China and South Korea declined to two digital numbers more than 40 days post $>100$ cases. Contrarily, the durations of many other countries post > 100 cases are already over 60 days by May 4, including France, Italy, Germany, Spain, US, UK and Iran, that the rapid increases of COVID-19 cases in those countries started at the end of February and beginning of March. Turkey and Russia have entered into their rapid increasing periods at middle of March. The epidemics of those two countries are still at high levels, but the duration times post > 100 cases are already 48 and 41 days. 
Compared with other 9 countries with the average of 30.4 days from > 100 cases to the top, the increase times of China and South Korea are much shorter ( 16 and 12 days). Moreover, the decrease times of China and South Korea are much longer than their increase times (about 1.9- to 2.5-fold longer), indicating that

it likely needs at least more than twice as long to get down to < 100 cases. Only 5 other countries (France, Germany, Italy, Spain and Iran) show the daily cases reduced to $-25 \%$ by May 4 , with the average of 39.4 days. If the decreasing speeds of those 5 countries were similar as China and South Korea, it still needs at least more than 20 days to get down to $<100$ cases per day. UK and Turkey reduced the daily cases to $-50 \%$ by May 4 , while USA and Russia are still at the high plateau. Obviously, it will take much longer time to get the daily cases down to less than 100 . The time of daily cases at high plateau ( $75 \%$ to $-75 \%)$ is another possible indicator for predicting the duration of the epidemic. The times of daily cases at high plateau of China ( 8 days) is shorter than that of 6 European countries and Iran (average of 16 days), and much shorter than that of USA ( 28 days). The longer duration at high plateau reflects larger numbers of the cases, which will definitely lead to longer decrease time.

We have also calculated the daily Rt values based on the daily reported COVID-19 cases each country. Notably, the Rt values of the analyzed countries have reduced to around 1.0 on the day with the largest number of cases, highlighting that the transmissibility of COVID-19 become relatively weak at the peak of prevalence. The long duration of decrease period is assumed to be correlated with the large amounts of cases that act as the sources of epidemic. In addition, the alteration of the daily Rt value seems to be related more closely with the changes of the ratios of daily cases, but not directly with the absolute numbers of daily cases in one country. Meanwhile, daily Rt value may also not directly associate with the durations of high plateau and whole time of the epidemic.

Certainly, the duration of COVID-19 epidemic is influenced by many factors, among which the strategies and measures of epidemic control play the essential role. Implementation of the control strategy and measure differs from different countries. It usually needs balance of the willingness of people, social system, the resources of public health, the feasibility of economic, etc. As the country that first reported COVID-19 epidemic, China has implemented the strictly tailored control measures that contribute greatly to shortening the duration time of epidemic definitely $(6,7)$.

The data presented here was collected from the websites of various governments, mainstream media, relevant professional websites, that may affect the accuracy and real-time performance. Deviations of the predictions and assessments from reality are probably inevitable.

\section{Conclusions}

The alteration of the daily Rt value seems to be related more closely with the changes of the ratios of daily cases, rather than the absolute numbers of daily cases in one country, meanwhile the implementation of strictly tailored control measures will greatly reduce the duration of the COVID-19 epidemic.

\section{Declarations}

\section{Ethical approval and consent to participate}

Not applicable.

\section{Consent for publication}

All authors have contributed to the information or material submitted for publication and have read and approved the manuscript.

\section{Availability of data and materials}

Not applicable.

\section{Competing interests}

The authors declare no conflict of interest.

\section{Funding}

This work was supported by National Key R\&D Program of China (2018YFC1200305).

\section{Author contributions}

CC and XPD designed the study and drafted the manuscript, CC and QS performed the statistical analysis. XPD conceived of the study, participated in its design and coordination and helped to draft the manuscript. All authors read and approved the final manuscript.

\section{Acknowledgements}

We thank Yidu Cloud (Beijing) Technology Co., Ltd. for their contributions to the article.

\section{Author's information}

State Key Laboratory for Infectious Disease Prevention and Control, NHC Key Laboratory of Medical Virology and Viral Diseases, Collaborative Innovation Center for Diagnosis and Treatment of Infectious Diseases (Zhejiang University), National Institute for Viral Disease Control and Prevention, Chinese Center for Disease Control and Prevention, Beijing, China

Cao Chen, Qi Shi, Xiao-Ping Dong 
Xiao-Ping Dong

Chinese Center for Disease Control and Prevention-Wuhan Institute of Virology, Chinese Academy of Sciences Joint Research Center for Emerging Infectious Diseases and Biosafety, Center for Biosafety Mega-Science, Chinese Academy of Sciences, Wuhan, China

Cao Chen, Xiao-Ping Dong

China Academy of Chinese Medical Sciences, Beijing, China

Xiao-Ping Dong

\section{References}

1. WHO. Coronavirus disease (COVID-19) United States: WHO; 2020 [.

2. COVID-19 coronavirus pandemic United States: Worldometers; 2020 [Available from: https://www.worldometers.info/coronavirus/country/us/.

3. The COVID Tracking Project United States: Data API; 2020 [Available from: https://covidtracking.com/api

4. Khan T, Agnihotri K, Tripathi A, Mukherjee S, Agnihotri N, Gupta G. COVID-19: A Worldwide, Zoonotic, Pandemic Outbreak. Altern Ther Health Med. 2020.

5. Poland GA. SARS-CoV-2: a time for clear and immediate action. Lancet Infect Dis. 2020;20(5):531-2.

6. Huang Y, Wu Q, Wang P, Xu Y, Wang L, Zhao Y, et al. Measures Undertaken in China to Avoid COVID-19 Infection: Internet-Based, Cross-Sectional Survey Study. J Med Internet Res. 2020;22(5):e18718.

7. Prem K, Liu Y, Russell TW, Kucharski AJ, Eggo RM, Davies N, et al. The effect of control strategies to reduce social mixing on outcomes of the COVID-19 epidemic in Wuhan, China: a modelling study. Lancet Public Health. 2020;5(5):e261-e70.

\section{Figures}

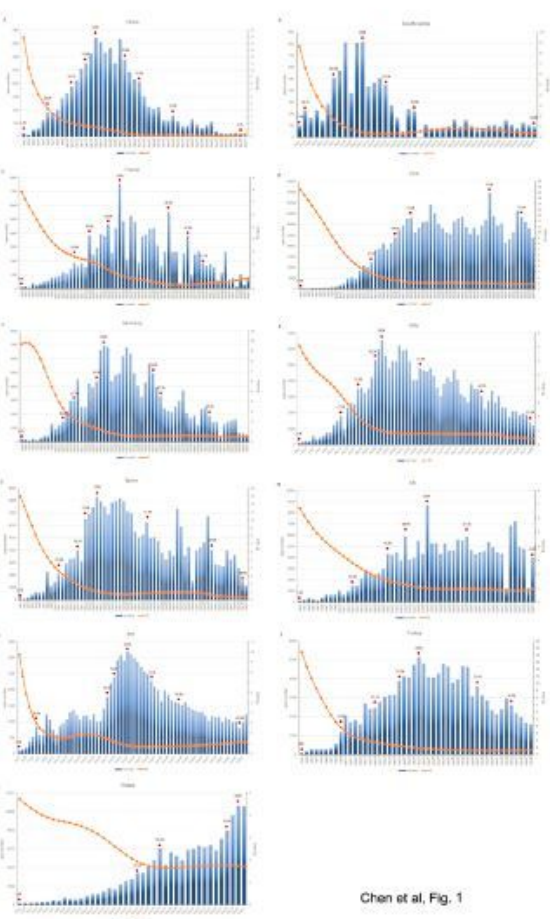

Figure 1

The daily new confirmed cases and Rt values of COVID-19 of 11 enrolled countries. The date from more than 100 cases to the date with less than 100 cases or by May 4, 2020. The case numbers are indicated on the left and presented as blue bar, roughly $25 \%, 50 \%, 75 \%$ and $100 \%$ are marked using red solid circle on the corresponding blue bars. The Rt values are indicated on the right and presented as orange solid line. Various countries are marked on the top each histogram (A-K). 


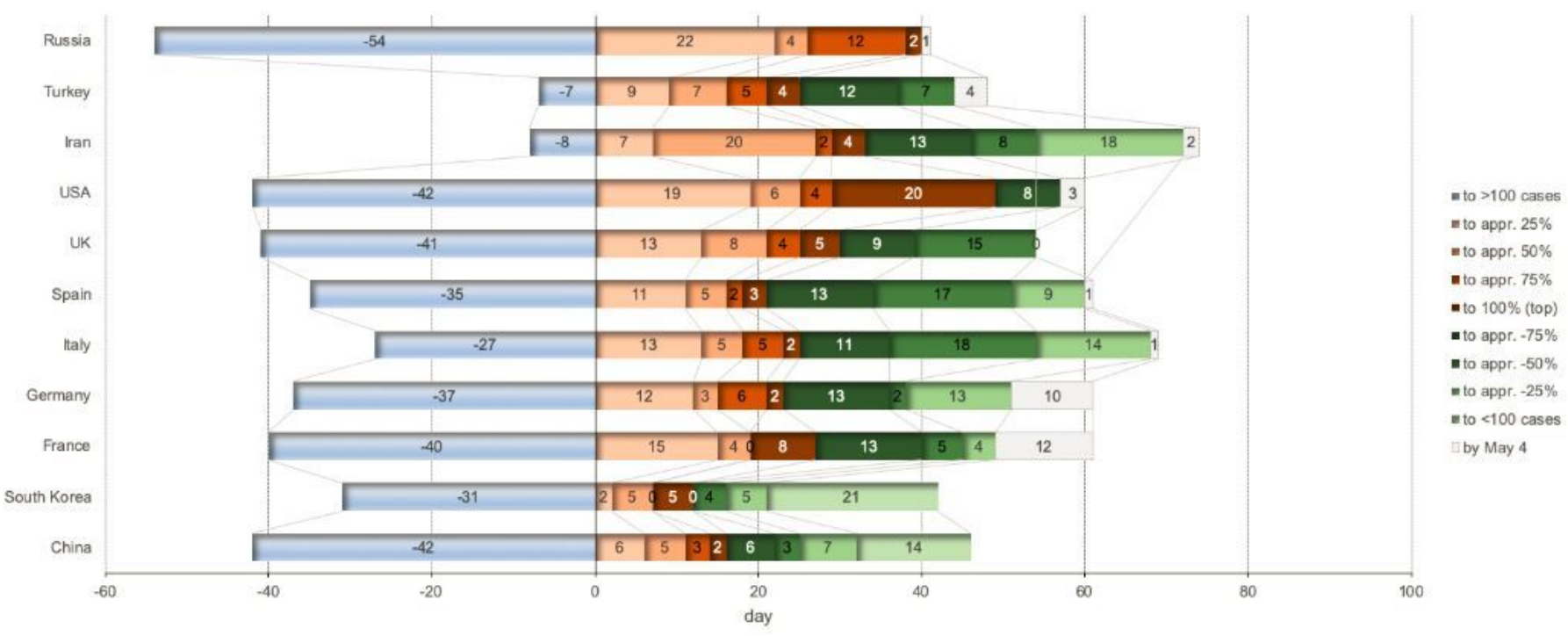

Figure 2

Duration times in the increase and decrease period based on the daily case ratio. Various duration times are marked in the histogram with different colors and illustrated on the right. $Y$ axis presents enrolled countries and $X$ axis indicated the interval time (day). 